\title{
Unstable manifolds of relative equilibria in Hamiltonian systems with dissipation*
}

\author{
Gianne Derks \\ Department of Mathematics and Statistics, \\ University of Surrey, Guildford, Surrey, GU2 5XH, UK \\ Tudor Ratiu ${ }^{\dagger}$ \\ Département de Mathématiques, \\ Ecole Polytechnique Fédérale de Lausanne, CH 1015 Lausanne, Switzerland
}

14 January 2002

\begin{abstract}
This paper studies the destabilizing effects of dissipation on families of relative equilibria in Hamiltonian systems which are non-extremal constraint critical points in the energy-Casimir or the energy-momentum methods. The dissipation is allowed to destroy the conservation law associated with the symmetry group or Casimirs, as long as the family of relative equilibria stays on an invariant manifold. This approach complements earlier work in the literature, in which the dissipation did not affect the conservation law.

First, Chetaev's instability theorem is extended to invariant manifolds and this extended theorem is used to prove instability of families of relative equilibria for several examples. Second, it is shown that families of non-extremal stationary solutions of the two-dimensional incompressible homogeneous Euler equations are unstable for the corresponding viscous perturbations of this system, that is, for the two-dimensional NavierStokes equations. Also, the instability of the sleeping top relative equilibria under friction can easily be proved in this way, even before the Hamiltonian sleeping top becomes linearly unstable. Finally, sufficient conditions are given for which friction destabilizes families of non-extremal relative equilibria in simple mechanical systems with abelian symmetry.
\end{abstract}

Keywords: Dissipation induced instability, invariant manifolds, Navier-Stokes, sleeping top

AMS classification scheme numbers: 58F30, 76D05, 70E50

\footnotetext{
${ }^{*}$ This work was partially supported by European Commission funding for the Research Training Network "Mechanics and Symmetry in Europe" (MASIE)

${ }^{\dagger}$ Research partially supported by the Swiss NSF
} 


\section{Introduction}

This paper considers destabilizing effects of dissipation on families of relative equilibria in Hamiltonian systems, so called dissipation induced instability. Relative equilibria can be found in Hamiltonian systems which are invariant under the action of a continuous symmetry group. In such systems, relative equilibria are solutions which move with constant speed along the flow of the symmetry group. For example, in a system with rotational symmetry the relative equilibria are uniformly rotating states.

Noether's theorem implies that continuous symmetries in Hamiltonian systems are related to functionals which are constants of motion in a Hamiltonian system (e.g. the angular momentum in case of a rotational symmetry). This observation leads to a variational description for the relative equilibria. Relative equilibria can be described as constrained critical points of the Hamiltonian on level sets of the constants of motion. Some Hamiltonian systems have constants of motion which are not related to continuous symmetries, but which are due to a degeneracy in the Poisson bracket. These constants of motion which generate the center of the Poisson algebra are called Casimirs and equilibrium solutions of Hamiltonian systems which are constrained critical points of the Hamiltonian on level sets of the Casimirs are also called relative equilibria. Usually, relative equilibria appear in families and the individual relative equilibria in such a family can be parametrized by the value of the constants of the motion.

The variational characterization of the relative equilibria can be used to get sufficient criteria for non-linear (orbital) stability. This has been formalized in two related methods. The first one is the energy-Casimir method; see Holm, Marsden, Ratiu \& Weinstein [13] for details and more references. The second method is the energy-momentum method, which does not rely on the existence of Casimir functions. For details and more references, see Simo, Lewis \& Marsden [18] and Simo, Posbergh \& Marsden [19]. These methods imply that if the relative equilibrium is a constrained extremum, then the relative equilibrium will be nonlinearly (orbitally) stable. However, no conclusions can be drawn if the relative equilibrium is a non-extremal constrained critical point. With spectral methods one can try to obtain some information about linear instability. However, it is hard to get any information about the nonlinear stability in case of a linearly stable (i.e., neutrally stable) relative equilibrium, which is a non-extremal constrained critical point.

If dissipation is added to a Hamiltonian system, in general it can not be expected that the functionals related to the symmetry group or Casimirs (constants of motion for the Hamiltonian case) remain invariant under the new dynamics. The dissipation will induce an evolution along the level sets. In addition, it is very unlikely that the orbits of individual relative equilibria are invariant under the dynamics of the dissipative system. However, since the manifold formed by the orbits of the family of relative equilibria includes various level sets of these functionals, it is possible that the full family of relative equilibria forms an invariant manifold under the dynamics of the system defined by the sum of the original Hamiltonian system and a dissipative vector field.

If the family of relative equilibria consists of extremal constrained equilibria, then dissipation will induce attractor-like properties for the family and a rate of attraction can be found. Under appropriate conditions, it can be shown that solutions starting near the family of relative equilibria will continue to stay near this family and will be asymptotically attracted to it. In DeRks, Lewis \& RATIU [6], the variational description of the relative equilibria and ideas 
from the energy-momentum method are used to develop a general theory in case of simple mechanical systems with dissipation. An illustration is given for a rigid body with friction. In DeRks \& VAN Groesen [7] a rate of attraction is found for the family of KdV cnoidal waves when uniform damping is added to the KdV equation. Attractors and an attraction rate are found for 2D Navier-Stokes and reduced magnetohydrodynamics in DERKS \& RATIU [8]. To derive the above mentioned stability results it is not necessary that the manifold of relative equilibria of the underlying Hamiltonian system is invariant under the dissipative vector field.

The present paper focuses on families of relative equilibria which are non-extremal constrained critical points and shows how dissipation can destabilize such families. Although the extremal families did not have to be invariant to have attractive properties under the influence of dissipation, the non-extremal cases turn out to be more delicate. It is often assumed that these families remain invariant after the addition of dissipation. In BLOCH, KRISHNAPRASAD, MARSDEn \& RATIU [3, 4] dissipation induced instability is shown for relative equilibria in Hamiltonian systems with a dissipation which does not destroy the conservation law associated with the symmetry group. In [3], the influence of so called internal dissipation on a relative equilibrium in a simple mechanical system with symmetry is considered. The block-diagonalization version of the energy-momentum method is used to strengthen results of Chetaev [5] and HAHn [12] and to prove both Lyapunov and spectral instability for those relative equilibria. In [4], the influence of group (or rotational) dissipation is studied in EulerPoincaré equations with double bracket dissipation. In both papers $[3,4]$ it is essential that the conservation law associated with the symmetry group is not destroyed after the addition of dissipation. In this paper this assumption will be relaxed to the hypothesis that the dissipation leaves the family invariant, but that the orbits of the individual relative equilibria do not have to be themselves invariant. Thus, instability will be shown for the invariant manifolds of relative equilibria, as opposed to that for individual relative equilibria as in $[3,4]$.

The paper is structured in the following way. In section 2, Chetaev's instability theorem for equilibrium points is extended to invariant manifolds. For the relative equilibria, a natural Lyapunov function is given by combining the Hamiltonian and Casimirs and/or other functionals related to the symmetry group. With the extended version of Chetaev's theorem, the analysis of the influence of dissipation on this Lyapunov function gives sufficient conditions for instability. These ideas are applied to various examples in the following sections.

In section 3, invariant manifolds in the two-dimensional Navier-Stokes equations with small viscosity and no forcing are considered. Without viscosity, the Navier-Stokes equations turn into the 2D Euler equations. The 2D Euler equations are Hamiltonian and have a large family of Casimirs, the enstrophy being one of them. The relative equilibria generated by combining the enstrophy and the Hamiltonian form manifolds of stationary solutions. Although the solutions are not stationary anymore if viscosity is turned on, the manifolds are still invariant. In section 3 it will be shown that the non-extremal manifolds are unstable under the dynamics of the Navier equations. These observations complement the work of FOIAS \& SAUT [10] where it is shown that every solution of the Navier-Stokes equations will asymptotically converge to one of the manifolds of relative equilibria; in addition, the first terms of the asymptotic expansion are derived. It is an open question which manifold of relative equilibria will be selected and this paper shows that not all solutions nearby a specific manifold will converge to it. Our work also refines observations in van GroesEn [11], where the instability of larger manifolds, which include the families of relative equilibria, is shown. 
In section 4, the influence of friction on the sleeping top is considered. A full analysis of the existence and stability of frictionless sleeping top relative equilibria can be found in Lewis, Ratiu, Simo \& Marsden [15]. It turns out that the sleeping top has several families of relative equilibria, which are non-extremal in the energy-momentum characterization but whose linearization is neutrally stable. As is known from experiments, these relative equilibria become unstable if friction acts on them. The analysis in this paper will show that the instability starts at values of the angular velocity for which the Hamiltonian sleeping top is linearly stable.

In the last section of examples, a complement to the dissipation induced instability of [3] is given for simple mechanical systems with symmetry. We will give sufficient conditions under which a dissipative perturbation leads to instability for families of non-extremal relative equilibria if the dissipation does not respect the conservation laws induced by the symmetries, but leaves the families invariant. The paper finishes with some concluding remarks and further problems.

\section{Instability criteria for invariant manifolds}

Consider the dynamical system

$$
u_{t}=f(u), \quad t>0,
$$

with $u$ in some manifold $\mathcal{H}$. Let $\boldsymbol{M}$ be an invariant manifold of (1). If $\mathcal{H}$ is a subset of a metric space $(\mathcal{X}, d)$, then define the $d$-distance between $u \in \mathcal{H}$ and $\boldsymbol{M}$ as

$$
d(u, \boldsymbol{M})=\inf _{v \in \boldsymbol{M}} d(u, v) .
$$

We will prove two lemmas which give sufficient conditions for the instability of the manifold $\boldsymbol{M}$. The lemmas are based on Chetaev's theorems for instability of equilibria, see e.g. Hahn [12], theorems 42.6, 42.7, Malkin [16], §13 and §15, and ZubOv [21], §1.13. An overview can be found in MERKIN [17].

For the first lemma we assume that $\left(\mathcal{X}_{1}, d_{1}\right)$ and $\left(\mathcal{X}_{2}, d_{2}\right)$ are two metric spaces which both contain $\mathcal{H}\left(\mathcal{X}_{1}=\mathcal{X}_{2}\right.$ and $d_{1}=d_{2}$ is possible $)$, i.e., $d_{1}$ and $d_{2}$ give two distances on $\mathcal{H}$.

Lemma 1 Let $L: \mathcal{H} \rightarrow \mathbb{R}$ be a differentiable functional, which satisfies:

1. There is some $\delta_{0}$ such that for all $0<\delta<\delta_{0}$ and for all $\hat{u} \in M$ there is some $u \in \mathcal{H}$ such that $d_{1}(u, \hat{u})<\delta$ and $L(u)<0$.

2. There is some $\varepsilon_{0}>0$ and some $C>0$ such that $L(u)>-C$, if $d_{2}(u, M)<\varepsilon_{0}$.

3. There is some $\varepsilon_{1}>0$ and some $\alpha>0$ such that

$$
\begin{gathered}
\langle D L(u), f(u)\rangle \leq \alpha L(u), \\
\text { if } d_{2}(u, \boldsymbol{M})<\varepsilon_{1} \text { and } L(u)<0 .
\end{gathered}
$$

Then $\boldsymbol{M}$ is an unstable manifold. To be explicit, there is some $\varepsilon>0$ such that for all $\delta>0$ and for all $\hat{u} \in \boldsymbol{M}$ there is some $u_{0} \in \mathcal{H}$ with $d_{1}\left(u_{0}, \hat{u}\right)<\delta$ and some $t_{0}>0$ such that the solution $u(t)$ of (1) with initial condition $u(0)=u_{0}$ satisfies $d_{2}\left(u\left(t_{0}\right), \boldsymbol{M}\right)>\varepsilon$. 
Remark 1 We can replace condition 2 by the following (similar) condition:

2a. There is some $\varepsilon_{0}>0$ such that for any solution $u(t)$ with $d_{1}(u(0), \boldsymbol{M})<\varepsilon_{0}$ or $d_{2}(u(0), \boldsymbol{M})<\varepsilon_{0}$ there is some $C>0$ such that $L(u(t))>-C$ for $t \geq 0$.

Proof We will prove this lemma by a contradiction argument. Assume that the manifold $\boldsymbol{M}$ is locally stable. Hence for all $\varepsilon>0$ there is some $\delta(\varepsilon)>0$ and some $\hat{u} \in \boldsymbol{M}$ such that for all $u_{0} \in \mathcal{H}$ with $d_{1}\left(u_{0}, \hat{u}\right)<\delta(\varepsilon)$ the solution $u(t)$ of (1) with initial condition $u(0)=u_{0}$ satisfies $d_{2}(u(t), \boldsymbol{M})<\varepsilon$, for all $t \geq 0$.

Take some $\varepsilon$ such that $0<\varepsilon<\min \left(\varepsilon_{0}, \varepsilon_{1}\right)$. Take some $u_{0} \in \mathcal{H}$ such that $d_{1}\left(u_{0}, \hat{u}\right)<\delta(\varepsilon)$ and $L\left(u_{0}\right)<0$. (We can do this because of condition 1.) Let $u(t)$ be the solution of (1) with initial condition $u_{0}$. Because $\boldsymbol{M}$ is locally stable, we have $d_{2}(u(t), \boldsymbol{M})<\varepsilon<\varepsilon_{1}$ for all $t \geq 0$.

Assume that there is some $T>0$ such that $L(u(t))<0$, for $0 \leq t<T$ and $L(u(T))=0$. Then we have

$$
0=L(u(T))=\int_{0}^{T} \frac{\mathrm{d}}{\mathrm{d} t} L(u(t)) d t+L(u(0))<\alpha \int_{0}^{T} L(u(t)) d t+0<0 .
$$

However this is a contradiction, hence for all $t \geq 0, L(u(t))<0$.

With condition 3 this implies for all $t \geq 0$

$$
\frac{\mathrm{d}}{\mathrm{d} t}\left(L(u(t)) e^{-\alpha t}\right) \leq 0 .
$$

Therefore, $L(u(t)) \leq e^{\alpha t} L\left(u_{0}\right)$ for all $t \geq 0$. Since $L\left(u_{0}\right)<0$, there is some $T>0$ such that $L(u(T))<-C$. But this contradicts condition 2 since $d_{2}(u(t), \boldsymbol{M})<\varepsilon<\varepsilon_{0}$. Hence the manifold $M$ is not locally stable.

Condition 3 of Lemma 1 implies that $L(u)=0$ if $u \in \boldsymbol{M}$. In the next lemma we will show that this condition can be relaxed to $\langle D L(u), f(u)\rangle<0$ if some extra conditions regarding completeness are satisfied. So we will restrict to finite dimensional manifolds $\mathcal{H}$.

Lemma 2 Let $\mathcal{H}$ be a manifold in a finite dimensional vector space and let $d$ be a distance on $\mathcal{H}$. Let $L: \mathcal{H} \rightarrow \mathbb{R}$ be a differentiable function which satisfies:

1. For all $u \in \boldsymbol{M}$ one has $L(u)=0$.

2. There is some $\delta_{0}>0$ such that for all $0<\delta<\delta_{0}$ and for all $\hat{u} \in \boldsymbol{M}$ there is some $u \in \mathcal{H}$ such that $d(u, \hat{u})<\delta$ and $L(u)<0$.

3. There is some $\varepsilon_{0}>0$ and some $C>0$ such that $d(u, \boldsymbol{M})<\varepsilon_{0}$ implies $L(u) \geq-C$.

4. For all $u \in \mathcal{H}$ with $L(u)<0$, we have

$$
\langle D L(u), f(u)\rangle<0
$$

if $0<d(u, \boldsymbol{M})<\varepsilon_{0}$.

If $\boldsymbol{M}$ is bounded or any solution near $\boldsymbol{M}$ is bounded, (i.e., if $\hat{u}$ is an arbitrary element of $\boldsymbol{M}$, then for every $u_{0} \in \mathcal{H}$ with $d\left(u_{0}, \boldsymbol{M}\right)<\varepsilon_{0}$ there is some $C_{1}\left(u_{0}\right)$ such that $d(u(t), \hat{u})<$ $C_{1}\left(u_{0}\right)$, for all $\left.t \geq 0\right)$, then $\boldsymbol{M}$ is an unstable manifold. To be explicit, there is some $\varepsilon>0$ such that for all $\delta>0$ and for all $\hat{u} \in \boldsymbol{M}$ there is some $u_{0} \in \mathcal{H}$ with $d\left(u_{0}, \hat{u}\right)<\delta$ and some $t_{0}>0$ such that the solution $u(t)$ of (1) with initial condition $u(0)=u_{0}$ satisfies $d\left(u\left(t_{0}\right), \boldsymbol{M}\right)>\varepsilon$. 
Remark 2 We can replace condition 3 by the following (similar) condition:

3a. For any solution $u(t)$ of (1), there is some $C>0$ such that $L(u(t)) \geq-C$ if $t \geq 0$.

Proof We will prove this lemma by a contradiction argument. Assume that the manifold $\boldsymbol{M}$ is locally stable. Hence for all $\varepsilon>0$ there is some $\delta(\varepsilon)>0$ and $\hat{u} \in \boldsymbol{M}$ such that for all $u_{0} \in \mathcal{H}$ with $d\left(u_{0}, \hat{u}\right)<\delta$ and for all $t>0$ the solution $u(t)$ of (1) with initial condition $u(0)=u_{0}$ satisfies $d(u(t), \boldsymbol{M})<\varepsilon$.

Take some $\varepsilon$ such that $0<\varepsilon<\varepsilon_{0}$. Take some $u_{0} \in \mathcal{H}$ such that $d\left(u_{0}, \hat{u}\right)<\delta(\varepsilon)$ and $L\left(u_{0}\right)<0$. (We can do this because of condition 2.) Let $u(t)$ be the solution of (1) with initial condition $u_{0}$. Because $\boldsymbol{M}$ is locally stable, we have $d(u(t), \boldsymbol{M})<\varepsilon<\varepsilon_{0}$ for all $t \geq 0$. Assume that there is some $T>0$ such that $L(u(t))<0$, for $0 \leq t<T$ and $L(u(T)=0$. With condition 4 it follows that

$$
0=L(u(T))=\int_{0}^{T} \frac{\mathrm{d}}{\mathrm{d} t} L(u(t)) d t+L(u(0))<0+0 .
$$

However this is a contradiction, hence for all $t \geq 0, L(u(t))<0$. Again with condition 4 this implies that for all $t>0$

$$
L(u(t))=L\left(u_{0}\right)+\int_{0}^{t} \frac{\mathrm{d}}{\mathrm{d} \tau} L(u(\tau)) d \tau<L\left(u_{0}\right) .
$$

Define the set $S_{1}$ by

$$
S_{1}=\left\{u \in \mathcal{H} \mid L(u)<L\left(u_{0}\right)\right\} .
$$

This means that there is some $C_{1}>0$ such that $d(u, M) \geq C_{1}$, for $u \in \overline{S_{1}}$. Indeed, assume that there is a sequence $\left\{u_{n}\right\} \subset S_{1}$ such that $d\left(u_{n}, \boldsymbol{M}\right) \rightarrow 0$. Since $L$ is continuous, this means that $L\left(u_{n}\right) \rightarrow L(\boldsymbol{M})=0$. But this contradicts the fact that $L\left(u_{n}\right)<L\left(u_{0}\right)<0$.

Define $-\ell=\inf \{\langle D L(u(t)), f(u(t))\rangle \mid t \geq 0\}$. We will show that $-\ell<0$. Let $t_{n}$ be a sequence such that $\left\langle D L\left(u\left(t_{n}\right)\right), f\left(u\left(t_{n}\right)\right)\right\rangle \rightarrow-\ell$. If $\boldsymbol{M}$ is bounded, then $d\left(u\left(t_{n}\right), \boldsymbol{M}\right)<\varepsilon_{0}$ implies that $\left\{u\left(t_{n}\right)\right\}$ is a bounded sequence. If $\boldsymbol{M}$ is not bounded, then by the assumption of the lemma, we have that $u\left(t_{n}\right)$ is a bounded sequence.

Since $u\left(t_{n}\right)$ is a bounded sequence, there is some convergent subsequence $u\left(t_{n_{k}}\right)$, such that $u\left(t_{n_{k}}\right) \rightarrow \hat{u}$. Since $u(t) \in \overline{S_{1}}$ for all $t \geq 0$, we know that $C_{1} \leq d(u(t), \boldsymbol{M}) \leq \varepsilon$. Hence, $0<C_{1} \leq d(\hat{u}, \boldsymbol{M}) \leq \varepsilon<\varepsilon_{0}$. Using condition 4 , we see that $-\ell=\langle D L(\hat{u}), f(\hat{u})\rangle<0$.

This means that $\frac{\mathrm{d}}{\mathrm{d} t} L(u(t)) \leq-\ell<0$ for all $t \geq 0$. Hence we can improve the estimate (2):

$$
L(u(t))=L\left(u_{0}\right)+\int_{0}^{t} \frac{\mathrm{d}}{\mathrm{d} \tau} L(u(\tau)) d \tau \leq L\left(u_{0}\right)-\int_{0}^{t} \ell d \tau=L\left(u_{0}\right)-\ell t
$$

But this means that $L(u(t))$ is unbounded from below, which contradicts condition 3 . Therefore $\boldsymbol{M}$ is unstable.

In the following sections these lemmas will be used to show that dissipation induces instability in non-extremal manifolds of relative equilibria. 


\section{Unstable manifolds in 2D Navier-Stokes}

Consider the two dimensional Navier-Stokes equations without forcing

$$
\begin{array}{ll}
\dot{\omega}=-\boldsymbol{v} \cdot \nabla \omega+\nu \Delta \omega=\{\psi, \omega\}+\nu \Delta \omega & \text { in } \mathcal{D} ; \\
\psi=0 \quad \text { and } \quad \omega=0, & \text { on } \partial \mathcal{D},
\end{array}
$$

where $\mathcal{D}$ is any compact, simply connected domain in the $x y$-plane with smooth boundary $\partial \mathcal{D}$ and $\psi$ is the stream function, i.e., $-\Delta \psi=\omega$.

If viscosity is absent, that is $\nu=0$ hence an ideal fluid is considered, then the NavierStokes equations become the Euler equations. The Euler equations form a Hamiltonian system relative to the total energy

$$
H(\omega)=\frac{1}{2} \int_{\mathcal{D}}|\boldsymbol{v}|^{2} d \boldsymbol{x}=\frac{1}{2} \int_{\mathcal{D}} \omega \psi d x .
$$

The Poisson structure for these equations is highly degenerate and, as a consequence, the 2D Euler equations have many Casimirs. For example, each functional depending on $\omega$ only is a Casimir. In particular, the enstrophy

$$
W(\omega)=\frac{1}{2} \int_{\mathcal{D}} \omega^{2} d \boldsymbol{x}
$$

is a Casimir for the Euler equations. It is easily verified that the critical points of the enstrophy $W$ on level sets of the Hamiltonian $H$ are stationary solutions of the Euler equations which satisfy

$$
-\Delta \omega=\lambda \omega, \quad \text { implying } \omega=\lambda \psi,
$$

where $\lambda$ is a positive eigenvalue of $(-\Delta)$ with zero boundary conditions. These solutions are a two-dimensional version of the Arnold-Beltrami-Childress flows (ABC) (cf. Dombre et al. [9]). In particular, if $\mathcal{D}$ is a disc, then these solutions are necessarily axi-symmetric. In the literature, sometimes the ABC flows are also called Stokes flows, since they are solutions of the Stokes operator.

Let $0<\lambda_{0}<\lambda_{1}<\ldots$ be the eigenvalues of $(-\Delta)$ with zero boundary conditions. Let $\mathcal{E}_{k}$ be the eigenspace with the eigenvalue $\lambda_{k}$, let $\Pi_{k}$ be the $L^{2}$-orthogonal projection onto $\mathcal{E}_{k}$, and let $\Pi_{k}^{\perp}$ be the projection on the orthogonal complement $\mathcal{E}_{k}^{\perp}$ of $\mathcal{E}_{k}$. Then for each eigenvalue $\lambda_{k}$, the Euler equations have a family of relative equilibria $\mathcal{E}_{k}$. Each relative equilibrium in $\mathcal{E}_{k}$ is a critical point of the functional $L_{k}(\omega)=W(\omega)-\lambda_{k} H(\omega)$ and for each relative equilibrium $\bar{\omega} \in \mathcal{E}_{k}, L_{k}(\bar{\omega})=0$. The functional $L_{0}$ is positive definite and vanishes on $\mathcal{E}_{0}$ only. Indeed, for each vorticity $\omega$ with stream function $\psi$ one has

$$
L_{0}(\omega)=\int_{\mathcal{D}} \omega\left(\omega-\lambda_{0} \psi\right) d \boldsymbol{x} \geq \frac{\lambda_{1}-\lambda_{0}}{\lambda_{1}}\left\|\Pi_{0}^{\perp} \omega\right\|_{L^{2}}^{2} .
$$

The energy-Casimir method shows that the manifold $\mathcal{E}_{0}$ is stable under the evolution of the Euler equations. On the other hand, the functionals $L_{k}$ are indefinite for $k \geq 1$, since if $\bar{\omega} \in \mathcal{E}_{0}$, then $L_{k}(\bar{\omega})=\left(\lambda_{0}-\lambda_{k}\right) H(\bar{\omega})<0$ and if $\bar{\omega} \in \mathcal{E}_{k+1}$, then $L_{k}(\bar{\omega})=\left(\lambda_{k+1}-\lambda_{k}\right) H(\bar{\omega})>0$. So the families $\mathcal{E}_{k}$ are non-extremal if $k \geq 1$ and nothing can be said about the stability of these manifolds with methods like the energy-Casimir method. 
Now we return to the full Navier-Stokes equations. The individual relative equilibria are not invariant anymore. It is straightforward to verify that for any $k \in \mathbb{N}$ and any $\omega_{\lambda_{k}} \in \mathcal{E}_{k}$

$$
\hat{\omega}(t)=e^{-\nu \lambda_{k} t} \omega_{\lambda_{k}}, \quad t \geq 0
$$

determines a solution of the Navier-Stokes equations. By global existence and uniqueness of the classical solutions for the 2D Navier-Stokes equations (see [20], section 2.2), these are all the solutions of the Navier-Stokes equations which at some $t_{0}$ (and hence for all $t$ ) lie in $\mathcal{E}_{k}$. Thus if $\hat{\omega}(0)=\omega_{\lambda_{k}}$ is some $\mathrm{ABC}-$ flow, at any other subsequent time according to the dynamics of the Navier-Stokes equations, $\hat{\omega}(t)$ is proportional to $\omega_{\lambda_{k}}$, the proportionality constant $e^{-\nu \lambda_{k} t}$ depending on time. Thus the $\mathrm{ABC}$-flows in $\mathcal{E}_{k}$ form invariant families under the dynamics of the Navier-Stokes equations.

Note also that by uniqueness of classical solutions, if $\omega_{0} \neq 0$, then the solution $\omega(t)$ of the Navier-Stokes equations with initial condition $\omega(0)=\omega_{0}$ satisfies also $\omega(t) \neq 0$ for all $t \geq 0$ (as a map from $[0, \infty)$ to the space of functions on the domain $\mathcal{D}$, not as a function of $(t, \boldsymbol{x}))$. In particular, $H(\omega(t)) \neq 0$ and $W(\omega(t)) \neq 0$.

In Derks \& RATiU [8] it is shown that the manifold $\mathcal{E}_{0}$ is an attractor for the NavierStokes equations: If $\omega(t)$ is a solution of the Navier-Stokes equations with $\omega(0)=\omega_{0}$ and $\left\|\Pi_{0}^{\perp} \omega_{0}\right\|^{2}<\frac{\lambda_{2}\left(\lambda_{1}-\lambda_{0}\right)}{\lambda_{0}\left(\lambda_{2}-\lambda_{1}\right)}\left\|\Pi_{0} \omega_{0}\right\|^{2}$, then

$$
\left\|\Pi_{0}^{\perp} \omega(t)\right\| \leq C\left(\omega_{0}\right) e^{-\nu\left(\lambda_{1}-\lambda_{0}\right) t}\left\|\Pi_{0} \omega(t)\right\|,
$$

for all $t \geq 0$. In this section it will shown that all the other manifolds $\mathcal{E}_{k}, k \geq 1$ are unstable.

Theorem 3 For all $k \geq 1$, the manifolds $\mathcal{E}_{k}$ are unstable under the flow of the Navier-Stokes equations. This means that there is some $\varepsilon>0$ such that for all $\delta>0$ and all $\hat{\omega} \in \mathcal{E}_{k}$ there is some $\omega_{0}$ with $\left\|\omega_{0}-\hat{\omega}\right\|<\delta\left\|\psi_{0}\right\|$ and some $t_{0}>0$ such that the solution $\omega(t)$ with initial condition $\omega(0)=\omega_{0}$ satisfies $\left\|\Pi_{k}^{\perp} \omega\left(t_{0}\right)\right\|>\varepsilon\left\|\nabla \psi\left(t_{0}\right)\right\|$. Recall that $\psi$ denotes the stream function.

Note that the stability and instability criteria are expressed in relative terms $\left(\frac{\left\|\Pi_{0}^{\perp} \omega(t)\right\|}{\left\|\Pi_{0} \omega(t)\right\|}\right.$ and $\frac{\left\|\Pi_{k}^{\perp} \omega\left(t_{0}\right)\right\|}{\left\|\nabla \psi\left(t_{0}\right)\right\|}$ respectively). This is due to the fact that the zero state is in each manifold $\mathcal{E}_{k}$, $k \geq 0$, and the zero state is a global attractor for the Navier-Stokes equations. In Figure 1, there is a sketch to visualize the basin of attraction and the instability region.

In [10] and [11] it is shown that for any solution $\omega(t)$ of the Navier-Stokes equation, the limit $\lim _{t \rightarrow \infty} \frac{W(\omega(t))}{H(\omega(t))}$ exists and equals some eigenvalue of $(-\Delta)$. From [10] it follows that the asymptotic expansion of $\omega(t)$ will start with an element of the set $\mathcal{E}_{k}$ corresponding to the asymptotic eigenvalue. Theorem 3 shows that this does not imply that the sets $\mathcal{E}_{k}$ are stable. There exist states, arbitrarily close to $\mathcal{E}_{k}$, which do not converge to $\mathcal{E}_{k}$. Furthermore, in [11] it is shown that the sets

$$
\mathcal{I}_{k}=\left\{\omega(0) \mid \lim _{t \rightarrow \infty} \frac{W(\omega(t))}{H(\omega(t))}=\lambda_{k}\right\}
$$

are not attracting if $k \geq 1$. It is shown that for each $\omega(0) \in \mathcal{I}_{k}$ there are (arbitrary small) perturbations $\xi$ and some $T>0$ such that $\omega(T)+\xi \notin \mathcal{I}_{k}$. However, the sets $\mathcal{I}_{k}$ are much larger and much more complicated than the manifolds $\mathcal{E}_{k}$. Also, no results about distances to $\mathcal{I}_{k}$ are shown in [11]. 


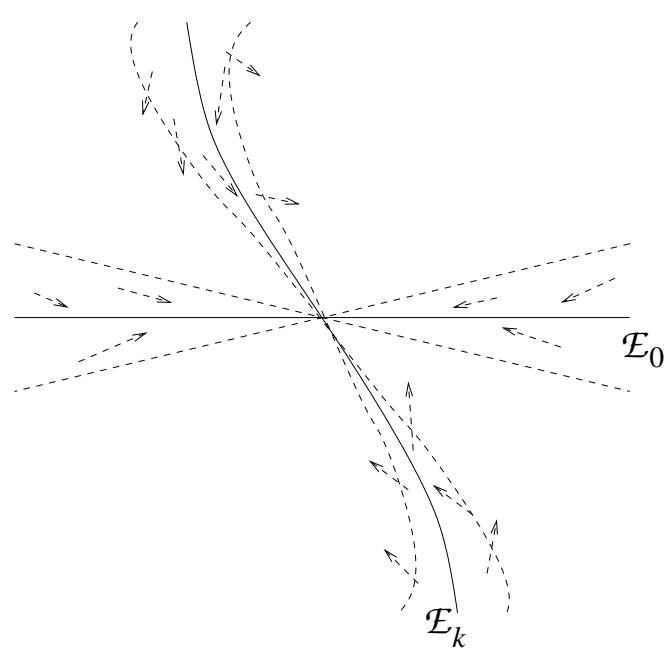

Figure 1: Sketch of the attracting manifold $\mathcal{E}_{0}$ with its basin of attraction and an unstable manifold $\mathcal{E}_{k}, k \geq 1$ with its region of instability.

Theorem 3 will be proved by using Lemma 1. Define the scaled Lyapunov function

$$
\hat{L}_{k}(\omega)=\frac{L_{k}(\omega)}{H(\omega)}=\frac{W(\omega)-\lambda_{k} H(\omega)}{H(\omega)} .
$$

Let $\omega(t)$ be a solution of the Navier-Stokes equation. Using the boundary condition $\left.\omega\right|_{\partial \mathcal{D}}=0$, the time derivatives of the Hamiltonian and the enstrophy functional are given by

$$
\begin{aligned}
& \frac{\mathrm{d}}{\mathrm{d} t} H(\omega(t))=-\nu \int_{\mathcal{D}} \omega^{2} d \boldsymbol{x}-\nu \int_{\partial D} \omega \frac{\partial \psi}{\partial n}=-2 \nu W(\omega(t)) \\
& \frac{\mathrm{d}}{\mathrm{d} t} W(\omega(t))=-\nu \int_{\mathcal{D}} \omega(-\Delta \omega) d \boldsymbol{x}=-\nu \int_{\mathcal{D}}|\nabla \omega|^{2} d \boldsymbol{x}-\nu \int_{\partial D} \omega \frac{\partial \omega}{\partial n}=-\nu \int_{\mathcal{D}}|\nabla \omega|^{2} d \boldsymbol{x} .
\end{aligned}
$$

From this, we get

$$
\frac{\mathrm{d}}{\mathrm{d} t} L_{k}(\omega(t))=-\nu \int_{\mathcal{D}}|\nabla \omega|^{2} d \boldsymbol{x}+\lambda_{k} \nu \int_{\mathcal{D}} \omega^{2} d \boldsymbol{x}=-\nu \int_{\mathcal{D}} \omega\left(-\Delta-\lambda_{k}\right) \omega d \boldsymbol{x}
$$

and

$$
\frac{\mathrm{d}}{\mathrm{d} t} \hat{L}_{k}(\omega(t))=2 \nu \hat{L}_{k}^{2}(\omega)+2 \nu \lambda_{k} \hat{L}_{k}(\omega)-\frac{\nu}{H(\omega)} \int_{\mathcal{D}} \omega\left(-\Delta-\lambda_{k}\right) \omega d \boldsymbol{x}=: G_{k}(\omega)
$$

see also DeRKs \& RATIU [8] for these computations.

We can estimate the integral in both expressions by using $L_{k}$.

Lemma 4 For any $\lambda$ with $\lambda_{k-1} \leq \lambda \leq \lambda_{k+1}$, one has

$$
\int_{\mathcal{D}} \omega\left(-\Delta-\lambda_{k}\right) \omega d \boldsymbol{x} \geq 2 \lambda L_{k}(\omega) .
$$

Proof Let $\mathbb{P}_{k}$ denote the $L^{2}$-orthogonal projection onto the first $k$ eigenspaces of $(-\Delta)$, i.e., onto $\mathcal{E}_{0} \oplus \ldots \oplus \mathcal{E}_{k-1}$. Then we can write

$$
\int_{\mathcal{D}} \omega\left(-\Delta-\lambda_{k}\right) \omega d \boldsymbol{x}=\int_{\mathcal{D}} \mathbb{P}_{k} \omega\left(-\Delta-\lambda_{k}\right) \mathbb{P}_{k} \omega d \boldsymbol{x}+\int_{\mathcal{D}} \mathbb{P}_{k}^{\perp} \omega\left(-\Delta-\lambda_{k}\right) \mathbb{P}_{k}^{\perp} \omega d \boldsymbol{x} .
$$


A Poincaré-like inequality gives

$$
\int_{\mathcal{D}} \mathbb{P}_{k}^{\perp} \omega\left(-\Delta-\lambda_{k}\right) \mathbb{P}_{k}^{\perp} \omega d \boldsymbol{x} \geq \lambda_{k+1} \int_{\mathcal{D}} \mathbb{P}_{k}^{\perp} \omega\left(-\Delta-\lambda_{k}\right) \mathbb{P}_{k}^{\perp} \psi d \boldsymbol{x}
$$

and

$$
\begin{aligned}
\int_{\mathcal{D}} \mathbb{P}_{k} \omega\left(\lambda_{k}-(-\Delta)\right) \mathbb{P}_{k} \omega d \boldsymbol{x} & \leq \lambda_{k-1} \int_{\mathcal{D}} \mathbb{P}_{k} \omega\left(\lambda_{k}-(-\Delta)\right) \mathbb{P}_{k} \psi d \boldsymbol{x} \\
& \leq \lambda \int_{\mathcal{D}} \mathbb{P}_{k} \omega\left(\lambda_{k}-(-\Delta)\right) \mathbb{P}_{k} \psi d \boldsymbol{x}
\end{aligned}
$$

for any $\lambda \geq \lambda_{k-1}$, since the integrals involved are non negative. So for any $\lambda \geq \lambda_{k-1}$ we can conclude

$$
\int_{\mathcal{D}} \mathbb{P}_{k} \omega\left(-\Delta-\lambda_{k}\right) \mathbb{P}_{k} \omega d \boldsymbol{x} \geq \lambda \int_{\mathcal{D}} \omega\left(-\Delta-\lambda_{k}\right) \psi d \boldsymbol{x}-\lambda \int_{\mathcal{D}} \mathbb{P}_{k}^{\perp} \omega\left(-\Delta-\lambda_{k}\right) \mathbb{P}_{k}^{\perp} \psi d \boldsymbol{x} .
$$

Substitution of this inequality and (8) into (7) gives

$$
\begin{aligned}
\int_{\mathcal{D}} \omega\left(-\Delta-\lambda_{k}\right) \omega d \boldsymbol{x} & \geq \lambda \int_{\mathcal{D}} \omega\left(-\Delta-\lambda_{k}\right) \psi d \boldsymbol{x}+\left(\lambda_{k+1}-\lambda\right) \int_{\mathcal{D}} \mathbb{P}_{k}^{\perp} \omega\left(-\Delta-\lambda_{k}\right) \mathbb{P}_{k}^{\perp} \psi d \boldsymbol{x} \\
& \geq 2 \lambda L_{k}(\omega),
\end{aligned}
$$

if $\lambda \leq \lambda_{k+1}$, since $\int_{\mathcal{D}} \mathbb{P}_{k}^{\perp} \omega\left(-\Delta-\lambda_{k}\right) \mathbb{P}_{k}^{\perp} \omega d \boldsymbol{x} \geq 0$, by definition of the operator $\mathbb{P}_{k}^{\perp}$.

Remark 3 The estimate in this lemma is sharp. To see this, consider the solution $\omega(t)=$ $e^{-\nu \lambda_{k-1} t} \hat{\omega}_{k-1}$, where $\hat{\omega}_{k-1} \in \mathcal{E}_{k-1}$. Then $L_{k}(\omega(t))=e^{-2 \nu \lambda_{k-1} t} L_{k}\left(\omega_{k-1}\right)$, hence $\frac{\mathrm{d}}{\mathrm{d} t} L_{k}(\omega(t))=$ $-2 \nu \lambda_{k-1} L_{k}(\omega(t))$. Similarly, for $\hat{\omega}_{k+1} \in \mathcal{E}_{k+1}$ and $\omega(t)=e^{-\nu \lambda_{k-1} t} \hat{\omega}_{k-1}$, it follows that $L_{k}(\omega(t))=e^{-2 \nu \lambda_{k+1} t} L_{k}\left(\omega_{k+1}\right)$, hence $\frac{\mathrm{d}}{\mathrm{d} t} L_{k}(\omega(t))=-2 \nu \lambda_{k+1} L_{k}(\omega(t))$.

Note that this also implies that the functional $L_{k}$ can not be used to apply Lemma 1. Indeed, since $L_{k}\left(\hat{\omega}_{k-1}\right)=\left(1-\frac{\lambda_{k}}{\lambda_{k-1}}\right) W\left(\hat{\omega}_{k-1}\right)<0$ this implies that there is no $\alpha>0$ such that $\frac{\mathrm{d}}{\mathrm{d} t} L_{k}(\omega(t)) \leq \alpha L_{k}(\omega(t))$. Moreover, $\frac{\mathrm{d}}{\mathrm{d} t} L_{k}(\omega(t))>0$ !

Using Lemma 4 with $\lambda=\lambda_{k-1}$ in the expression for $\frac{\mathrm{d}}{\mathrm{d} t} \hat{L}_{k}(\omega)=G_{k}(\omega)$, equality (6) gives

$$
G_{k}(\omega) \leq 2 \nu \hat{L}_{k}(\omega)\left(\hat{L}_{k}(\omega)+\lambda_{k}-\lambda_{k-1}\right)
$$

It will be shown that this implies that if $\omega$ is near $\mathcal{E}_{k}$ and $L_{k}(\omega)<0$, then there is some $\alpha>0$ such that $G_{k}(\omega) \leq 2 \nu \alpha \hat{L}_{k}(\omega)$.

First define a "scaled distance" to $\mathcal{E}_{k}$ as

$$
d\left(\omega, \mathcal{E}_{k}\right)^{2}=\min _{v \in \mathcal{E}_{k}} \frac{W(\omega-v)}{H(\omega)}=\min _{v \in \mathcal{E}_{k}} \frac{\|\omega-v\|^{2}}{\|\nabla \psi\|^{2}},
$$

with $\|\cdot\|$ the $L^{2}$ norm and $\omega=-\Delta \psi$. Writing $\omega=\Pi_{k} \omega+\Pi_{k}^{\perp} \omega$, we see that

$$
H(\omega) d\left(\omega, \mathcal{E}_{k}\right)^{2}=\min _{v \in \mathcal{E}_{k}} \frac{1}{2}\left(\left\|\Pi_{k} \omega-v\right\|^{2}+\left\|\Pi_{k}^{\perp} \omega\right\|^{2}\right)=\frac{1}{2}\left\|\omega-\Pi_{k} \omega\right\|^{2} .
$$

And

$$
\left\|\omega-\Pi_{k} \omega\right\|^{2}=\|\omega\|^{2}+\left\|\Pi_{k} \omega\right\|^{2}-2\left\langle\left(\Pi_{k} \omega+\Pi_{k}^{\perp} \omega\right), \Pi_{k} \omega\right\rangle_{L^{2}}=\|\omega\|^{2}-\left\|\Pi_{k} \omega\right\|^{2} .
$$


So altogether we get

$$
d\left(\omega, \mathcal{E}_{k}\right)^{2}=\frac{\left\|\omega-\Pi_{k} \omega\right\|^{2}}{2 H(\omega)}=\frac{W(\omega)-W\left(\Pi_{k} \omega\right)}{H(\omega)} .
$$

Next we derive an upper and a lower bound on $\hat{L}_{k}(\omega)$ in terms of the distance $d\left(\omega, \mathcal{E}_{k}\right)$. Write

$$
\begin{aligned}
L_{k}(\omega) & =W(\omega)-\lambda_{k} H(\omega)=W(\omega)-\lambda_{k} H\left(\Pi_{k} \omega\right)+\lambda_{k} H\left(\Pi_{k} \omega\right)-\lambda_{k} H(\omega) \\
& =W(\omega)-W\left(\Pi_{k} \omega\right)+\lambda_{k}\left(H\left(\Pi_{k} \omega\right)-H(\omega)\right)=d\left(\omega, \mathcal{E}_{k}\right)^{2} H(\omega)-\lambda_{k}\left(H(\omega)-H\left(\Pi_{k} \omega\right)\right) .
\end{aligned}
$$

Clearly $\left(H(\omega)-H\left(\Pi_{k} \omega\right)\right) \geq 0$, hence

$$
L_{k}(\omega) \leq d\left(\omega, \mathcal{E}_{k}\right)^{2} H(\omega) \quad \text { and } \quad \hat{L}_{k}(\omega) \leq d\left(\omega, \mathcal{E}_{k}\right)^{2} .
$$

Furthermore, since $\int_{\mathcal{D}} P_{k}^{\perp} \omega\left(-\Delta-\lambda_{k}\right) P_{k}^{\perp} \psi d \boldsymbol{x} \geq 0$, and a Poincaré inequality gives that $\int_{\mathcal{D}}\left(P_{k} \omega\right)\left(P_{k} \psi\right) d \boldsymbol{x} \leq \frac{1}{\lambda_{0}} \int_{\mathcal{D}}\left(P_{k} \omega\right)^{2} d \boldsymbol{x}$, we have

$$
\begin{aligned}
L_{k}(\omega) & =\frac{1}{2} \int_{\mathcal{D}} \omega\left(-\Delta-\lambda_{k}\right) \psi d \boldsymbol{x} \\
& =\frac{1}{2} \int_{\mathcal{D}} P_{k} \omega\left(-\Delta-\lambda_{k}\right) P_{k} \psi d \boldsymbol{x}+\frac{1}{2} \int_{\mathcal{D}} P_{k}^{\perp} \omega\left(-\Delta-\lambda_{k}\right) P_{k}^{\perp} \psi d \boldsymbol{x} \\
& \geq-\frac{1}{2} \int_{\mathcal{D}} P_{k} \omega\left(\lambda_{k}-(-\Delta)\right) P_{k} \psi d \boldsymbol{x} \geq-\frac{\lambda_{k}-\lambda_{0}}{2 \lambda_{0}} \int_{\mathcal{D}}\left(P_{k} \omega\right)^{2} d \boldsymbol{x} \\
& \geq-\frac{\lambda_{k}-\lambda_{0}}{\lambda_{0}}\left(W(\omega)-W\left(\Pi_{k} \omega\right)\right)=-\frac{\lambda_{k}-\lambda_{0}}{\lambda_{0}} d\left(\omega, \mathcal{E}_{k}\right)^{2} H(\omega) .
\end{aligned}
$$

Summarizing, the following lemma is proved.

Lemma 5 For all $\omega$ one has

$$
-\frac{\lambda_{k}-\lambda_{0}}{\lambda_{0}} d\left(\omega, \mathcal{E}_{k}\right)^{2} \leq \hat{L}_{k}(\omega) \leq d\left(\omega, \mathcal{E}_{k}\right)^{2}
$$

Now we are ready to prove an estimate on $G_{k}$. Define $\beta=\frac{\lambda_{0}\left(\lambda_{k}-\lambda_{k-1}\right)}{\lambda_{k}-\lambda_{0}}$.

Lemma 6 For all $0<\varepsilon<1$, all $\omega$ such that $d\left(\omega, \mathcal{E}_{k}\right)^{2}<\beta(1-\varepsilon)$ and $\hat{L}_{k}(\omega)<0$, satisfy

$$
G_{k}(\omega) \leq 2 \nu \varepsilon\left(\lambda_{k}-\lambda_{k-1}\right) \hat{L}_{k}(\omega) .
$$

Proof Let $0<\varepsilon<1$. Let $\omega$ be such that $d\left(\omega, \mathcal{E}_{k}\right)^{2}<\beta(1-\varepsilon)$. From (10) it follows

$$
\hat{L}_{k}(\omega) \geq-\frac{\lambda_{k}-\lambda_{0}}{\lambda_{0}} \beta(1-\varepsilon)=-\left(\lambda_{k}-\lambda_{k-1}\right)(1-\varepsilon) .
$$

If $\hat{L}_{k}(\omega)<0$, then we get by substitution of this equality into (9)

$$
G_{k}(\omega) \leq 2 \nu \hat{L}_{k}(\omega)\left(\lambda_{k}-\lambda_{k-1}\right)(1-(1-\varepsilon))=2 \nu \varepsilon\left(\lambda_{k}-\lambda_{k-1}\right) \hat{L}_{k}(\omega) .
$$

So now Lemma 1 can be applied with $L=\hat{L}_{k}$ to prove Theorem 3 .

Proof of Theorem 3 It will be shown that $\hat{L}_{k}$ satisfies the conditions of Lemma 1. 
1. Let $\hat{\omega}_{0} \in \mathcal{E}_{0},\left\|\hat{\omega}_{0}\right\|=1$. For all $\eta \in \mathbb{R}$ and $\hat{\omega} \in \mathcal{E}_{k}$, one has $\hat{L}_{k}\left(\hat{\omega}+\eta \omega_{0}\|\hat{\omega}\|\right)=$ $\frac{\eta^{2}\left(\lambda_{0}-\lambda_{k}\right) \lambda_{k}}{\lambda_{0}+\eta^{2} \lambda_{k}}<0$.

2. Since $W(\omega) \geq \lambda_{0} H(\omega)$, it follows that $\hat{L}_{k}(\omega) \geq\left(\lambda_{0}-\lambda_{k}\right)$ for all $\omega$.

3. Since $G_{k}(\omega)=\frac{\mathrm{d}}{\mathrm{d} t} \hat{L}_{k}(\omega(t))=\left\langle D \hat{L}_{k}(\omega), f(\omega)\right\rangle$, Lemma 4 shows that for all $\varepsilon_{1}<$ $\frac{\lambda_{0}\left(\lambda_{k}-\lambda_{k-1}\right)}{\lambda_{k}-\lambda_{0}}$ there is some $\alpha$ such that all $\omega$ with $\hat{L}_{k}(\omega)<0$ and $d\left(\omega, \mathcal{E}_{k}\right)<\varepsilon_{1}$ satisfy $\left\langle D \hat{L}_{k}(\omega), f(\omega)\right\rangle \leq \alpha \hat{L}_{k}(\omega)$.

Hence the conditions of Lemma 1 are satisfied and we can conclude that the manifolds $\mathcal{E}_{k}$ are unstable, i.e., there is some $\varepsilon>0$ such that for all $\delta>0$ and all $\hat{\omega} \in \mathcal{E}_{k}$ there is some $\omega_{0}$ with $d\left(\omega_{0}, \hat{\omega}\right)<\delta$ and some $t_{0}>0$ such that the solution $\omega(t)$ with initial condition $\omega(0)=\omega_{0}$ satisfies $d\left(\omega\left(t_{0}\right), \mathcal{E}_{k}\right)>\varepsilon$. By observing that $2 H(\omega)=\|\nabla \psi\|^{2}$, we get $d\left(\omega_{0}, \hat{\omega}\right)=\frac{\left\|\omega_{0}-\hat{\omega}\right\|}{\left\|\nabla \psi_{0}\right\|}$ and $d\left(\omega, \mathcal{E}_{k}\right)=\frac{\left\|\omega-\Pi_{k} \omega\right\|}{\|\nabla \psi\|}$, which leads to the formulation as presented in the Theorem.

\section{The sleeping top}

In this section the influence of friction on the motion of a sleeping asymmetric top will be investigated. A sleeping top is a rigid body with a fixed point in a gravitational field, rotating about the axis through the center of mass and the fixed point. An asymmetric top is a top which is not axially symmetric (for example, a Lagrange top is a rigid body which is axially symmetric). A detailed geometric description of the stability of sleeping tops without friction and bifurcations from sleeping tops can be found in LEwis et al. [15].

First we derive a suitable phase space to describe the motion of the rigid body. Since the rigid body has a fixed shape and a fixed point, the configuration space $Q$ is the set of all permissible orientations of the body. Choosing a reference orientation $\mathcal{B}$, the configuration $\mathcal{B}(t)$ at time $t$ can be described as

$$
\mathcal{B}(t)=\Lambda(t) \mathcal{B},
$$

where $\Lambda(t)$ is a uniquely determined element of the rotation group $S O(3)$. Let ${ }^{\wedge}$ denote the map from $\mathbb{R}^{3}$ to the space of skew-symmetric matrices such that for any $\boldsymbol{\xi} \in \mathbb{R}^{3}$, the skew-symmetric matrix $\widehat{\boldsymbol{\xi}}$ is determined by

$$
\widehat{\boldsymbol{\xi}} \boldsymbol{x}=\boldsymbol{\xi} \times \boldsymbol{x}, \text { for all } \boldsymbol{x} \in \mathbb{R}^{3} .
$$

The tangent space $T Q$ can be identified with $S O(3) \times \mathbb{R}^{3}$ in the following way. Let $(\Lambda, \delta \Lambda)$ be a pair in $T Q$, then there is a unique $\delta \Theta \in \mathbb{R}^{3}$ such that

$$
\delta \Lambda=\Lambda \widehat{\delta \Theta}
$$

This is the so called left trivialization of $T Q$ and the variables $(\Lambda, \widehat{\delta \boldsymbol{\Theta}})$ are called body variables. Similarly, the canonical phase space $T^{*} Q$ can be identified with $S O(3) \times \mathbb{R}^{3}$. If $\left(\Lambda, \delta \Pi_{\Lambda}\right)$ is a pair in $T^{*} Q$, then there is a unique $\Pi \in \mathbb{R}^{3}$ such that

$$
\delta \Pi_{\Lambda}=\Lambda \widehat{\Pi} .
$$

Furthermore, the covector-vector pairing in body coordinates becomes

$$
\left\langle\delta \Pi_{\Lambda}, \delta \Lambda\right\rangle=\frac{1}{2} \operatorname{tr}\left((\Lambda \widehat{\mathbf{\Pi}})^{T}(\Lambda \widehat{\delta \boldsymbol{\Theta}})\right)=\mathbf{\Pi} \cdot \delta \boldsymbol{\Theta} .
$$


As usual, the (reference/body) inertia tensor is defined by

$$
\left.\mathbb{I}=\int_{\mathcal{B}} \rho(\boldsymbol{X})\left(|\boldsymbol{X}|^{2}\right) \mathbf{1}_{3}-\boldsymbol{X} \otimes \boldsymbol{X}\right) d^{3} \boldsymbol{X}
$$

where $\mathbf{1}_{3}$ denotes the identity 3 by 3 matrix and $\rho$ is the mass density function of the rigid body. It is always possible to choose body coordinates, centered at the fixed point, such that the inertia tensor $\mathbb{I}=\operatorname{diag}\left(I_{1}, I_{2}, I_{3}\right)$. The 3 -axis will be taken to be the line connecting the fixed point and the center of mass and without loss of generality, we can choose the 1- and 2 -axis such that $I_{1} \geq I_{2}$. Furthermore, if

$$
m=\int_{\mathcal{B}} \rho(\boldsymbol{X}) d^{3} \boldsymbol{X}
$$

is the total mass and

$$
\boldsymbol{M}=\int_{\mathcal{B}} \rho(\boldsymbol{X}) \boldsymbol{X} d^{3} \boldsymbol{X}
$$

then $\frac{M}{m}$ is the center of mass. We will denote the length of the vector $\frac{M}{m}$ by $l$. The kinetic energy of the rigid body is $K(\boldsymbol{\Pi})=\frac{1}{2} \boldsymbol{\Pi} \cdot \mathbb{I}^{-1} \boldsymbol{\Pi}$ and the potential energy is $U(\Lambda)=g \Lambda \boldsymbol{M} \cdot \boldsymbol{e}_{3}$, where $\boldsymbol{e}_{3}$ is taken as the negative direction of the gravitational field and $g$ denotes the gravitational constant. So the Hamiltonian of the rigid body is

$$
H(\Lambda, \boldsymbol{\Pi})=\frac{1}{2} \boldsymbol{\Pi} \cdot \mathbb{I}^{-1} \boldsymbol{\Pi}+g \Lambda \boldsymbol{M} \cdot \boldsymbol{e}_{3} .
$$

Writing $\boldsymbol{U}=(\Lambda, \boldsymbol{\Pi})$, the equations of motion of the rigid body with friction are

$$
\dot{\boldsymbol{U}} \equiv J \nabla H(\boldsymbol{U})-\varepsilon P(\boldsymbol{U}), \quad \text { where } \quad J=\left(\begin{array}{cc}
\mathbf{0}_{3} & \mathbf{1}_{3} \\
-\mathbf{1}_{3} & \widehat{\boldsymbol{\Pi}}
\end{array}\right) \quad \text { and } \quad P(\boldsymbol{U})=\left(\begin{array}{c}
0 \\
\boldsymbol{\Pi}
\end{array}\right) .
$$

Here $\mathbf{1}_{3}$ is the identity 3 by 3 matrix, $\mathbf{0}_{3}$ is the zero 3 by 3 matrix, and $\varepsilon$ is the friction coefficient. Note that we have identified $\mathbb{I}^{-1} \boldsymbol{\Pi}$ with $\widehat{\Lambda \mathbb{I}^{-1} \mathbf{\Pi}}$. For a derivation of these equations, see for example ARNOLD [1].

Next we consider the symmetries of the system. The Hamiltonian is invariant under rotations about the vector $\boldsymbol{e}_{3}$ acting on the left of $\Lambda$. (Compared to the free rigid body, the gravity potential has broken the symmetry for the left rotations about the other axes.) Friction acts on the body angular momenta $\Pi$ only, so the system with dissipation is equivariant under these left rotations too. Since the rigid body is asymmetric, the maximal symmetry group is $G=S^{1}$. (If the rigid body is a Lagrange top, then there is also the symmetry of a rotation of the rigid body about its symmetry axis, hence in this case the symmetry group is $G=S^{1} \times S^{1}$.) The infinitesimal generator for a left rotation about the $\boldsymbol{e}_{3}$-axis with angular velocity $\xi$ is $X_{\xi}: T^{*} Q \rightarrow T\left(T^{*} Q\right)$.

$$
X_{\xi}(\Lambda, \boldsymbol{\Pi})=\left(\Lambda, \boldsymbol{\Pi} ; \xi \widehat{\boldsymbol{e}_{3}} \Lambda, 0\right) \equiv\left(\Lambda, \boldsymbol{\Pi} ; \xi \Lambda^{T} \boldsymbol{e}_{3}, 0\right) .
$$

So the momentum map (angular momentum) $\mathbb{J}: T^{*} Q \rightarrow \mathfrak{g}^{*} \equiv \mathbb{R}$ is given by

$$
\mathbb{J}(\Lambda, \boldsymbol{\Pi})=\Lambda^{T} \boldsymbol{e}_{3} \cdot \boldsymbol{\Pi} .
$$


Ignoring friction (i.e., taking $\varepsilon=0$ ), relative equilibria are the rigid body rotating with constant angular velocity about the $\boldsymbol{e}_{3}$-axis. In a moving frame, these solutions can be described as critical points of the Hamiltonian on level sets of the momentum map. The Euler-Lagrange equation for these critical points is

$$
D H(\boldsymbol{U})-\xi D J(\boldsymbol{U})=0, \quad \text { i.e., } \quad\left\{\begin{array}{c}
(g \widehat{\boldsymbol{M - \xi}} \boldsymbol{\Pi}) \Lambda^{T} \boldsymbol{e}_{3}=0 \\
\mathbb{I}^{-1} \boldsymbol{\Pi}-\xi \Lambda^{T} \boldsymbol{e}_{3}=0
\end{array} .\right.
$$

The Lagrange multiplier is the angular velocity $\xi$ of the rigid body. One solution of these equations is the asymmetric rigid body spinning about the axis passing through both the fixed point and $\boldsymbol{M}$ (the body 3 -axis), where this axis also coincides with the spatial $\boldsymbol{e}_{3}$ axis. This is the so called sleeping top motion, see Figure 4 for an illustration of this motion. If

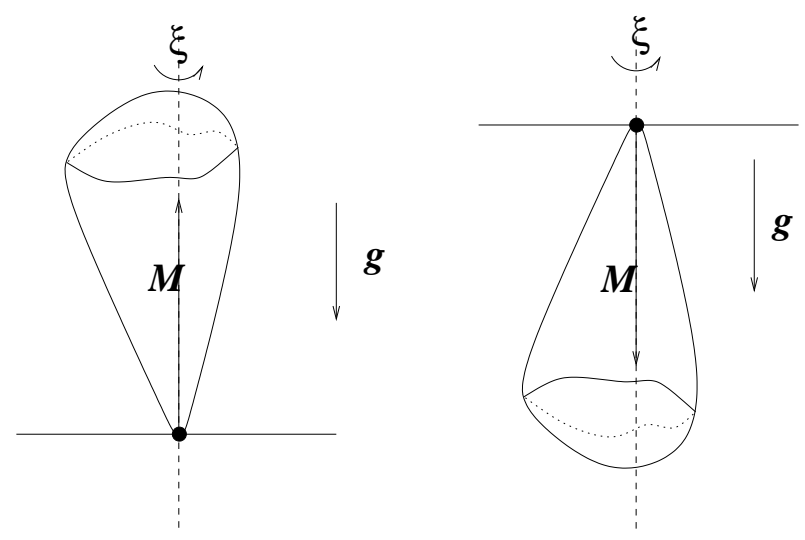

Figure 2: An example of an upright sleeping top is on the left and of and hanging sleeping top is on the right.

we denote the rotation by an angle $\theta$ about the $\boldsymbol{e}_{3}$-axis by $R_{3, \theta}$, then a relative equilibrium can be written as

$$
\overline{\boldsymbol{U}}=(\bar{\Lambda}, \overline{\boldsymbol{\Pi}})=\left(R_{3, \xi t+\theta_{0}}, \xi I_{3} \boldsymbol{e}_{3}\right) .
$$

The value of the momentum map at a relative equilibrium is $\mathbb{J}(\overline{\boldsymbol{U}})=\xi I_{3}$.

For the asymmetric top, the symmetry group consists of rotations about $\boldsymbol{e}_{3}$ and this group acts freely. A stability analysis for the sleeping top without friction can be found in [15]. The nonlinear stability analysis via the energy-momentum method gives that

- the upright sleeping top is nonlinearly stable if $I_{3}>I_{1}\left(\geq I_{2}\right)$ and its angular velocity $\xi$ satisfies $\xi^{2}>\frac{m g l}{I_{3}-I_{1}}$

- the hanging sleeping top is always nonlinearly stable if $I_{3}>I_{1}\left(\geq I_{2}\right)$;

- the hanging sleeping top is nonlinearly stable if $I_{3}<I_{1}$ and its angular velocity $\xi$ satisfies $\xi^{2}<\frac{m g l}{I_{1}-I_{3}}$.

These results are found by analyzing the second variation $D^{2} H_{\xi}(\overline{\boldsymbol{U}})$, where $H_{\xi}(\boldsymbol{U})=$ $H(\boldsymbol{U})-\xi \mathbb{J}(\boldsymbol{U})$ is the so called augmented Hamiltonian or energy-momentum function and $\overline{\boldsymbol{U}}$ 
is a solution of (11). If $D^{2} H_{\xi}(\overline{\boldsymbol{U}})$ is positive definite, then the relative equilibrium associated with $\overline{\boldsymbol{U}}$ is nonlinearly stable.

A linear stability analysis of the sleeping top can be found in [15] too. This analysis gives neutral stability for some sleeping tops which have an indefinite second variation $D^{2} H_{\xi}(\overline{\boldsymbol{U}})$. The parameter values of these sleeping tops are listed in Table 1.

$$
\begin{array}{c|c|c}
\hline & \text { Upright } & \text { Hanging } \\
\hline \hline I_{1} \geq I_{2} \geq I_{3} & \xi^{2}>\tau_{\mathrm{s}-\mathrm{f}}^{+} & \frac{m g l}{I_{2}-I_{3}}<\xi^{2} \\
I_{1}>I_{3}>I_{2} & \frac{m g l}{I_{3}-I_{2}}>\xi^{2}>\tau_{\mathrm{s}-\mathrm{f}}^{+} & \\
I_{3}>I_{1} \geq I_{2} & \frac{m g l}{I_{3}-I_{2}}>\xi^{2}>\tau_{\mathrm{s}-\mathrm{f}}^{+} & \\
\text {with } \tau_{\mathrm{s}-\mathrm{f}}^{+}=\frac{m g l\left(4 I_{1} I_{2}-I_{3}\left(I_{1}+I_{2}\right)+2 \sqrt{I_{1} I_{2}\left(2 I_{1}-I_{3}\right)\left(2 I_{2}-I_{3}\right)}\right)}{I_{3}^{2}\left(I_{1}+I_{2}-I_{3}\right)}
\end{array}
$$

Table 1: Parameter values for $I_{i}$ and $\xi$ for which a sleeping top with an indefinite augmented Hamiltonian is linearly stable.

We will show that for $I_{3}>I_{1} \geq I_{2}$, friction destabilizes the sleeping top relative equilibria which are linearly stable with an indefinite augmented Hamiltonian. First we analyze the effect of the friction on the behavior of the momentum map $\mathbb{J}$. Differentiation of the momentum map with respect to time gives

$$
\frac{\mathrm{d}}{\mathrm{d} t} \mathbb{J}(\boldsymbol{U})=D \mathbb{J}(\boldsymbol{U}) \cdot \dot{\boldsymbol{U}}=D \mathbb{J}(\boldsymbol{U}) \cdot J D H(\boldsymbol{U})-\varepsilon D \mathbb{J}(\boldsymbol{U}) \cdot P(\boldsymbol{U})=-\varepsilon \Lambda^{T} \boldsymbol{e}_{3} \cdot \boldsymbol{\Pi}=-\varepsilon \mathbb{J}(\boldsymbol{U}) .
$$

Hence for any solution $\boldsymbol{U}(t)$, the value of the momentum map is exponentially decaying: $\mathbb{J}(\boldsymbol{U}(t))=\mathbb{J}(\boldsymbol{U}(0)) e^{-\varepsilon t}$. The full family of sleeping top relative equilibria as described in (12) is invariant under fraction, although an individual sleeping top relative equilibrium is clearly not invariant. To be explicit, if one starts on the family of sleeping tops at the state $\left(R_{3, \theta_{0}}, \xi_{0} I_{3} \boldsymbol{e}_{3}\right)$, then the motion of the rigid body will be described by

$$
(\Lambda(t), \boldsymbol{\Pi}(t))=\left(R_{3, f(t)}, \xi_{0} e^{-\varepsilon t} I_{3} \boldsymbol{e}_{3}\right), \quad \text { with } \quad f(t)=\xi_{0} \frac{1-e^{-\varepsilon t}}{\varepsilon}+\theta_{0} .
$$

To analyze the stability of this type of motion on the family of sleeping tops, we define the Lyapunov function

$$
L(\boldsymbol{U})=H(\boldsymbol{U})-H(\overline{\boldsymbol{U}}), \quad \text { with } \overline{\boldsymbol{U}} \text { a sleeping top such that } \mathbb{J}(\overline{\boldsymbol{U}})=\mathbb{J}(\boldsymbol{U}) .
$$

Note that the Lyapunov function and the augmented Hamiltonian are closely related. Indeed, $L(\boldsymbol{U})=H_{\xi}(\boldsymbol{U})-H_{\xi}(\overline{\boldsymbol{U}})$, where $\xi=\frac{\mathbb{J}(\boldsymbol{U})}{I_{3}}$. If $\boldsymbol{U}(t)$ is a solution of the equation with friction, then

$$
\begin{aligned}
\frac{\mathrm{d}}{\mathrm{d} t} L(\boldsymbol{U}(t)) & =D H_{\xi}(\boldsymbol{U}) \cdot \dot{\boldsymbol{U}}-D H_{\xi}(\overline{\boldsymbol{U}}) \cdot \dot{\overline{\boldsymbol{U}}}=D H_{\xi}(\boldsymbol{U}) \cdot[J D H(\boldsymbol{U})-\varepsilon P(\boldsymbol{U})]-0 \\
& =-\varepsilon D H_{\xi}(\boldsymbol{U}) \cdot P(\boldsymbol{U})=-\varepsilon\left[\mathbb{I}^{-1} \boldsymbol{\Pi}-\xi \Lambda^{T} \boldsymbol{e}_{3}\right] \cdot \boldsymbol{\Pi} \\
& =-\varepsilon \boldsymbol{\Pi} \cdot \mathbb{I}^{-1} \boldsymbol{\Pi}+\varepsilon \xi \Lambda^{T} \boldsymbol{e}_{3} \cdot \boldsymbol{\Pi}=-\varepsilon \boldsymbol{\Pi} \cdot \mathbb{I}^{-1} \boldsymbol{\Pi}+\varepsilon \xi \mathbb{J}(\boldsymbol{U}) \\
& =-\varepsilon \boldsymbol{\Pi} \cdot \mathbb{I}^{-1} \boldsymbol{\Pi}+\varepsilon \xi^{2} I_{3}=-\varepsilon \boldsymbol{\Pi} \cdot \mathbb{I}^{-1} \boldsymbol{\Pi}+\varepsilon \frac{(\mathbb{J}(\boldsymbol{U}))^{2}}{I_{3}} .
\end{aligned}
$$


Nearby a sleeping top we can write $\Lambda=R_{3, \theta}+\Delta \Lambda$. There is some $\Delta \Theta \in \mathbb{R}^{3}$ and some symmetric matrix $\Delta S$, such that $\Delta \Lambda=R_{3, \theta}(\widehat{\Delta \Theta}+\Delta S)$. The rotation angle $\theta$ will be chosen such that $\Delta \Theta \cdot e_{3}=0$. Using that $\Lambda=R_{3, \theta}+\Delta \Lambda$ and $R_{3, \theta}$ are orthogonal matrices, we get

$$
\begin{aligned}
\mathbf{1}_{3} & =\left(R_{3, \theta}+\Delta \Lambda\right)^{T}\left(R_{3, \theta}+\Delta \Lambda\right)=\mathbf{1}_{3}+(\Delta \Lambda)^{T} \Lambda+\Lambda^{T}(\Delta \Lambda)+(\Delta \Lambda)^{T}(\Delta \Lambda) \\
& =\mathbf{1}_{3}+(-\widehat{\Delta \boldsymbol{\Theta}}+\Delta S)+(\widehat{\Delta \boldsymbol{\Theta}}+\Delta S)+(-\widehat{\Delta \boldsymbol{\Theta}}+\Delta S)(\widehat{\Delta \boldsymbol{\Theta}}+\Delta S) \\
& =\mathbf{1}_{3}+2 \Delta S-(\widehat{\Delta \boldsymbol{\Theta}})^{2}-\widehat{\Delta \boldsymbol{\Theta}} \Delta S+\Delta S \widehat{\Delta \boldsymbol{\Theta}}+(\Delta S)^{2} .
\end{aligned}
$$

Hence $\Delta S=\frac{1}{2}(\widehat{\Delta \boldsymbol{\Theta}})^{2}+\mathcal{O}\left(|\Delta \boldsymbol{\Theta}|^{4}\right)$ and $\Delta \Lambda=R_{3, \theta}\left(\widehat{\Delta \boldsymbol{\Theta}}+\frac{1}{2}(\widehat{\Delta \boldsymbol{\Theta}})^{2}\right)+\mathcal{O}\left(|\Delta \boldsymbol{\Theta}|^{4}\right)$.

Similarly, near a sleeping top we can write $\boldsymbol{\Pi}=\xi I_{3} \boldsymbol{e}_{3}+\delta \boldsymbol{\Pi}$, for some $\delta \boldsymbol{\Pi} \in \mathbb{R}^{3}$ and $\xi=\frac{\mathbb{J}(u)}{I_{3}}$. Using the definition $\mathbb{J}(u)=\Lambda^{T} \boldsymbol{e}_{3} \cdot \boldsymbol{\Pi}$ and $\delta \boldsymbol{U}=(\Delta \boldsymbol{\Theta}, \delta \boldsymbol{\Pi})$, this gives

$$
\begin{aligned}
\xi & =\frac{1}{I_{3}}\left(R_{3, \theta}\left(\mathbf{1}_{3}+\widehat{\Delta \boldsymbol{\Theta}}+\Delta S\right)\right)^{T} \boldsymbol{e}_{3} \cdot\left(\xi I_{3} \boldsymbol{e}_{3}+\delta \boldsymbol{\Pi}\right) \\
& =\xi+\frac{1}{I_{3}} \boldsymbol{e}_{3} \cdot \delta \boldsymbol{\Pi}-\xi \widehat{\Delta \boldsymbol{\Theta}} \boldsymbol{e}_{3} \cdot \boldsymbol{e}_{3}-\frac{1}{I_{3}} \widehat{\Delta \boldsymbol{\Theta}} \boldsymbol{e}_{3} \cdot \delta \boldsymbol{\Pi}+\xi \Delta S \boldsymbol{e}_{3} \cdot \boldsymbol{e}_{3}+\mathcal{O}\left(|\delta \boldsymbol{U}|^{3}\right) \\
& =\xi+\frac{1}{I_{3}} \boldsymbol{e}_{3} \cdot \delta \boldsymbol{\Pi}-\frac{1}{I_{3}} \widehat{\Delta \boldsymbol{\Theta}} \boldsymbol{e}_{3} \cdot \delta \boldsymbol{\Pi}+\frac{\xi}{2}(\widehat{\Delta \boldsymbol{\Theta}})^{2} \boldsymbol{e}_{3} \cdot \boldsymbol{e}_{3}+\mathcal{O}\left(|\delta \boldsymbol{U}|^{3}\right) .
\end{aligned}
$$

Hence

$$
\boldsymbol{e}_{3} \cdot \delta \boldsymbol{\Pi}=\widehat{\Delta \boldsymbol{\Theta}} \boldsymbol{e}_{3} \cdot \delta \mathbf{\Pi}-\frac{\xi}{2 I_{3}}(\widehat{\Delta \boldsymbol{\Theta}})^{2} \boldsymbol{e}_{3} \cdot \boldsymbol{e}_{3}+\mathcal{O}\left(|\delta \boldsymbol{U}|^{3}\right)=\mathcal{O}\left(|\delta \boldsymbol{U}|^{2}\right)
$$

Altogether, near the family of sleeping top relative equilibria, the expression for $\frac{\mathrm{d}}{\mathrm{d} t} L(\boldsymbol{U}(t))$ can be written as

$$
\begin{aligned}
-\varepsilon \boldsymbol{\Pi} \cdot \mathbb{I}^{-1} \boldsymbol{\Pi}+\varepsilon \frac{(\mathbb{J}(\boldsymbol{U}))^{2}}{I_{3}}= & -\varepsilon\left(\xi I_{3} \boldsymbol{e}_{3}+\delta \boldsymbol{\Pi}\right) \cdot \mathbb{I}^{-1}\left(\xi I_{3} \boldsymbol{e}_{3}+\delta \boldsymbol{\Pi}\right)+\frac{\varepsilon}{I_{3}}\left(\left(R_{3, \theta}+\Delta \Lambda\right)^{T} \boldsymbol{e}_{3} \cdot\left(\xi I_{3} \boldsymbol{e}_{3}+\delta \boldsymbol{\Pi}\right)\right)^{2} \\
= & -\varepsilon \xi^{2} I_{3}+\varepsilon \xi^{2} I_{3}-2 \varepsilon \xi \boldsymbol{e}_{3} \cdot \delta \boldsymbol{\Pi}+2 \varepsilon \xi \boldsymbol{e}_{3} \cdot \delta \boldsymbol{\Pi}+2 \varepsilon \xi^{2} I_{3}(\Delta \Lambda)^{T} \boldsymbol{e}_{3} \cdot \boldsymbol{e}_{3} \\
& -\varepsilon \delta \boldsymbol{\Pi} \cdot \mathbb{I}^{-1} \delta \boldsymbol{\Pi}+\frac{\varepsilon}{I_{3}}\left(\boldsymbol{e}_{3} \cdot \delta \boldsymbol{\Pi}\right)^{2}+\varepsilon \xi^{2} I_{3}\left((\Delta \Lambda)^{T} \boldsymbol{e}_{3} \cdot \boldsymbol{e}_{3}\right)^{2} \\
& +2 \varepsilon \xi(\Delta \Lambda)^{T} \boldsymbol{e}_{3} \cdot \delta \boldsymbol{\Pi}+2 \varepsilon \xi\left(\boldsymbol{e}_{3} \cdot \delta \boldsymbol{\Pi}\right)\left((\Delta \Lambda)^{T} \boldsymbol{e}_{3} \cdot \boldsymbol{e}_{3}\right)+\mathcal{O}\left(|\delta \boldsymbol{U}|^{3}\right) .
\end{aligned}
$$

Since $\Delta \Lambda=R_{3, \theta}\left(\widehat{\Delta \boldsymbol{\Theta}}+\frac{1}{2}(\widehat{\Delta \boldsymbol{\Theta}})^{2}\right)+\mathcal{O}\left(|\Delta \boldsymbol{\Theta}|^{4}\right)$, it follows that

$$
(\Delta \Lambda)^{T} \boldsymbol{e}_{3} \cdot \boldsymbol{e}_{3}=-\widehat{\Delta \boldsymbol{\Theta}} \boldsymbol{e}_{3} \cdot \boldsymbol{e}_{3}-\frac{1}{2}\left|\widehat{\Delta \boldsymbol{\Theta}} \boldsymbol{e}_{3}\right|^{2}+\mathcal{O}\left(|\Delta \boldsymbol{\Theta}|^{4}\right)=0-\frac{1}{2}\left|\widehat{\Delta \boldsymbol{\Theta}} \boldsymbol{e}_{3}\right|^{2}+\mathcal{O}\left(|\Delta \boldsymbol{\Theta}|^{4}\right) .
$$

Thus, near the family of sleeping top relative equilibria, for $I_{3}>I_{1}\left(\geq I_{2}\right)$ we have

$$
\begin{aligned}
\frac{\mathrm{d}}{\mathrm{d} t} L(\boldsymbol{U}(t)) & =-\varepsilon \xi^{2} I_{3}\left|\widehat{\Delta \boldsymbol{\Theta}} \boldsymbol{e}_{3}\right|^{2}-\varepsilon \delta \boldsymbol{\Pi} \cdot \mathbb{I}^{-1} \delta \boldsymbol{\Pi}-2 \varepsilon \xi \widehat{\Delta \boldsymbol{\Theta}} \boldsymbol{e}_{3} \cdot \delta \boldsymbol{\Pi}+\mathcal{O}\left(|\delta \boldsymbol{U}|^{3}\right) \\
& =-\frac{\varepsilon}{I_{3}}\left|I_{3} \xi \widehat{\Delta \boldsymbol{\Theta}} \boldsymbol{e}_{3}+\delta \boldsymbol{\Pi}\right|^{2}-\varepsilon \delta \boldsymbol{\Pi} \cdot\left(\mathbb{I}^{-1}-\frac{1}{I_{3}} \mathbf{1}_{3}\right) \delta \boldsymbol{\Pi}+\mathcal{O}\left(|\delta \boldsymbol{U}|^{3}\right) \\
& <0
\end{aligned}
$$

if $|\delta \boldsymbol{U}|$ is small, but unequal to zero, since $\Delta \boldsymbol{\Theta} \cdot \boldsymbol{e}_{3}=0$ by the choice of $\theta$ in $R_{3, \theta}$ and $\boldsymbol{e}_{3} \cdot \delta \boldsymbol{\Pi}=\mathcal{O}\left(|\delta \boldsymbol{U}|^{2}\right)$. By applying Lemma 2, we can conclude that friction destabilizes the neutrally stable upright sleeping tops with angular velocity $\frac{m g l}{I_{3}-I_{2}}>\xi^{2}>\tau_{\mathrm{s}-\mathrm{f}}^{+}$. 
Theorem 7 Consider an asymmetric top with its moments of inertia $I_{3}>I_{1} \geq I_{2}$. The upright sleeping top motion with angular velocity $\xi<\frac{m g l}{I_{3}-I_{1}}$ is unstable if friction acts on the top.

Proof Let $\boldsymbol{M}$ be the manifold of upright sleeping tops with angular velocity $\xi<\frac{m g l}{I_{3}-I_{1}}$. It will be shown that $L(\boldsymbol{U})$ satisfies the conditions of Lemma 2 .

1. By definition of $L$ and $\boldsymbol{M}, L(\boldsymbol{U})=0$ if $\boldsymbol{U} \in \boldsymbol{M}$.

2. In [15] it is shown that $L(\boldsymbol{U})$ is indefinite near the sleeping top relative equilibria if $\xi<\frac{m g l}{I_{3}-I_{1}}$.

3. Since $\boldsymbol{M}$ is bounded, $H(\boldsymbol{U})$ is bounded for $\boldsymbol{U}$ near $\boldsymbol{M}$ and we can conclude that $L(\boldsymbol{u})$ is bounded near $\boldsymbol{M}$.

4. From (13) it follows that $\langle D L(\boldsymbol{U}), f(\boldsymbol{U})\rangle<0$ near the family of sleeping tops.

Since $\xi<\frac{m g l}{I_{3}-I_{1}}$, the manifold $\boldsymbol{M}$ is bounded, hence with Lemma 2 it follows that $\boldsymbol{M}$ is unstable if friction is added.

Since $\frac{\mathrm{d}}{\mathrm{d} t} L(\boldsymbol{U}(t))<0$ if $\boldsymbol{U}(t)$ is near the family of sleeping top relative equilibria, also if $\xi^{2}>\frac{m g l}{I_{3}-I_{1}}$, it is easy to show that friction stabilizes the motion of the sleeping top if $\xi^{2}>\frac{m g l}{I_{3}-I_{1}}$. However, we have seen that friction causes the angular momentum to decay, hence the angular velocity $\xi$ will decay. Therefore, at some moment $\xi^{2}<\frac{m g l}{I_{3}-I_{1}}$ and friction will start to destabilize the motion, causing the usually observed collapse of the motion of an upright sleeping top.

\section{Instability caused by friction}

In this section we will give a first step towards a general approach to the stability and instability of relative equilibria in a symmetric classical mechanical system with friction. An overview of the stability analysis of an equilibrium in such system can be found in chapter 6 of Merkin [17]. The Thomson-Tait-Chetaev theorems are at the basis of this analysis. In BLOCH et al. [3, 4], a general approach to the stability of a relative equilibrium is given as long as the dissipation respects the invariance of the extra constant of motion that gave rise to the relative equilibrium. An important consequence of this assumption is that the relative equilibrium is a solution of the dissipative system too. In this section a more general type of dissipation is considered, which does not respect the invariance of the extra constant of motion that gave rise to the relative equilibria. However, it will be assumed that the family of relative equilibria (as a family, NOT as individual relative equilibria) is invariant. Then, with the use of Lemma 2, a dissipation induced instability theorem can be proved.

Consider a simple mechanical system with symmetry (see BLOCH et al. [3], or Simo et al. [18], or references in these papers). The Hamiltonian of such a system is given by

$$
\tilde{H}(\tilde{\boldsymbol{q}}, \tilde{\boldsymbol{p}})=\frac{1}{2}\|\tilde{\boldsymbol{p}}\|_{\tilde{q}}^{2}+V(\tilde{\boldsymbol{q}}),
$$

where $\tilde{\boldsymbol{q}}$ is in some configuration manifold $Q$ and $\tilde{\boldsymbol{p}} \in T_{\tilde{q}}^{*} Q$ is the conjugate momentum. It is assumed that the Hamiltonian $H$ is invariant under the action of a symmetry group $G$. 
For simplicity, we will assume that $G$ is abelian. Let $\mathbf{J}: T^{*} Q \rightarrow \mathfrak{g}^{*}$ be the momentum map. Assume that there is a manifold of relative equilibria, which can be parametrized by the value of the momentum map:

$$
\tilde{\mathcal{M}}=\left\{\left(\tilde{\boldsymbol{q}}_{e}(\mu), \tilde{\boldsymbol{p}}_{e}(\mu)\right) \mid \mu \in \mathfrak{g}^{*}\right\}
$$

As shown in $[3,18]$, the linearization around the manifold $\tilde{\mathcal{M}}$, using coordinates based on the energy-momentum method, leads to a gyroscopic system. Friction acts on the momenta only, so adding friction to such a system gives

$$
\begin{aligned}
\dot{\varphi} & =-\omega_{0}^{\prime}(\mu)-\frac{1}{2}\left[\left(\frac{\mathrm{d}}{\mathrm{d} \mu} M^{-1}(\mu) \boldsymbol{p}, \boldsymbol{p}\right)+\left(\Lambda^{\prime}(\mu) \boldsymbol{q}, \boldsymbol{q}\right)\right] \\
\dot{\mu} & =-\varepsilon f(\mu) \\
\dot{\boldsymbol{q}} & =M^{-1}(\mu) \boldsymbol{p} \\
\dot{\boldsymbol{p}} & =-\Lambda(\mu) \boldsymbol{q}-S M^{-1}(\mu) \boldsymbol{p}-\varepsilon R(\mu) M^{-1}(\mu) \boldsymbol{p} .
\end{aligned}
$$

Here $\mu$ is the value of the momentum map and $(\boldsymbol{q}, \boldsymbol{p})$ are the so called internal variables, i.e., they describe the non-group dynamics within the $\mathbf{J}^{-1}(\mu)$ level set. Finally $\varphi$ represent the coordinates of the group action, their dynamics does not influence the analysis of the stability of the manifold $\tilde{\mathcal{M}}$.

Also, $M, \Lambda$, and $R$ are symmetric and $S$ is skew-symmetric. Furthermore, $M$ and $R$ are strictly positive definite and $\Lambda$ is assumed to be invertible. (This last assumption is probably not essential, but there is not straightforward extension of the ideas in [3], since we need a $\mu$-dependent transformation to obtain the split of $\Lambda$ in an invertible part and a part which is zero.) For $\varepsilon=0$, the system is Hamiltonian with Hamiltonian $H$ and Poisson operator $\Gamma$ given by

$$
H(\mu, \boldsymbol{q}, \boldsymbol{p})=\omega_{0}(\mu)+\frac{1}{2}\left[\left(M^{-1}(\mu) \boldsymbol{p}, \boldsymbol{p}\right)+(\Lambda(\mu) \boldsymbol{q}, \boldsymbol{q})\right] \quad \text { and } \quad \Gamma=\left(\begin{array}{cccc}
0 & I & & \varnothing \\
-I & 0 & & \\
\varnothing & 0 & I \\
& -I & -S
\end{array}\right)
$$

In the new coordinates, the invariant manifold of relative equilibria is written as

$$
\mathcal{M}=\left\{(\mu, \varphi, \mathbf{0}, \mathbf{0}) \mid \mu \in \mathfrak{g}^{*}, \quad \varphi \in G\right\} .
$$

The system (14) is similar to a system analyzed by Chetaev [5], but with the extra feature of cyclic coordinates. A functional similar to the one in Chetaev's analysis can be used to prove stability or instability. Indeed, define

$$
L(\mu, \boldsymbol{q}, \boldsymbol{p})=H(\mu, \boldsymbol{q}, \boldsymbol{p})-H(\mu, \mathbf{0}, \mathbf{0})+\varepsilon \delta(\Lambda(\mu) \boldsymbol{q}, \boldsymbol{p}),
$$


for $\delta>0$ small. Then for $\delta$ small and $(\boldsymbol{q}, \boldsymbol{p}) \neq(\mathbf{0}, \mathbf{0})$

$$
\begin{aligned}
2 \frac{\mathrm{d}}{\mathrm{d} t} L & (\mu(t), \boldsymbol{q}(t), \boldsymbol{p}(t))= \\
& -\varepsilon\left(\left[2 R-\delta\left(2 M \Lambda+S^{2}-\varepsilon R^{2}-\varepsilon^{2} M D_{\mu} \Lambda \cdot f(\mu) M\right)\right] M^{-1} \boldsymbol{p}, M^{-1} \boldsymbol{p}\right) \\
& -\varepsilon \delta(1-\varepsilon)\|\Lambda \boldsymbol{q}\|^{2} \\
& -\varepsilon \delta\left\|\Lambda \boldsymbol{q}+S M^{-1} \boldsymbol{p}\right\|^{2}-\varepsilon^{2} \delta\left\|\Lambda \boldsymbol{q}+R M^{-1} \boldsymbol{p}\right\|^{2} \\
& -\varepsilon\left[\left(D_{\mu} M^{-1}(\mu) \cdot f(\mu) \boldsymbol{p}, \boldsymbol{p}\right)+\left(D_{\mu} \Lambda(\mu) \cdot f(\mu)(\boldsymbol{q}+\varepsilon \delta \boldsymbol{p}),(\boldsymbol{q}+\varepsilon \delta \boldsymbol{p})\right)\right] \\
& <0
\end{aligned}
$$

if $D_{\mu} \Lambda(\mu) \cdot f(\mu)$ and $D_{\mu} M^{-1}(\mu) \cdot f(\mu)$ are positive semi-definite.

By using Lemma 2 we get

Theorem 8 Consider the system (14). The manifold of relative equilibria is given by $\mathcal{M}=$ $\{(\mu, \varphi, \mathbf{0}, \mathbf{0})\}$ and is invariant under the dynamics. Assume that the matrices $D_{\mu} M^{-1}(\mu)$. $f(\mu) \geq 0, D_{\mu} \Lambda(\mu) \cdot f(\mu) \geq 0$ and that there is some $\bar{\lambda}_{0}>0$ such that for all $t \geq 0$ the eigenvalues of $\Lambda(\mu(t))$ are larger than or equal to $-\bar{\lambda}_{0}$.

- The manifold $\mathcal{M}$ is asymptotically stable if $\Lambda>0$.

- The manifold $\mathcal{M}$ is unstable if $\Lambda$ has negative eigenvalues.

Proof The Lyapunov function can be written as

$2 L(\mu, \boldsymbol{q}, \boldsymbol{p})=\left(\left[M^{-1}-\varepsilon^{2} \delta^{2} \Lambda\right] \boldsymbol{p}, \boldsymbol{p}\right)+(\Lambda[\boldsymbol{q}+\varepsilon \delta \boldsymbol{p}],[\boldsymbol{q}+\varepsilon \delta \boldsymbol{p}])$.

- The first observation in the theorem is an application of Lyapunov's stability theorem. Since $\Lambda$ is strictly positive definite, $L(\mu . \boldsymbol{q}, \boldsymbol{p})$ measures the distance from $(\mu, \boldsymbol{q}, \boldsymbol{p})$ to $\mathcal{M}$. The estimate on the time derivative of $L$ shows that this distance is decreasing.

- To verify the second observation, we check the conditions of Lemma 2 for the functional $L(\mu, \boldsymbol{q}, \boldsymbol{p})$ with $\delta$ small.

1. Clearly $L(\mathcal{M})=0$.

2. Since $\Lambda$ is indefinite, there is some $\boldsymbol{v}$ such that $(\Lambda \boldsymbol{v}, \boldsymbol{v})<0$. Hence $L(\mu, \boldsymbol{v}, \mathbf{0})=$ $(\Lambda \boldsymbol{v}, \boldsymbol{v})<0$ and $L$ has negative directions near $\mathcal{M}$.

3. Define $-\lambda_{0}(\mu)$ to be the smallest eigenvalue of $\Lambda(\mu)$. Since $\Lambda$ is indefinite, $-\lambda_{0}<$ 0 . If $d((\mu, \boldsymbol{q}, \boldsymbol{p}), \mathcal{M})<\sqrt{\varepsilon_{0}}$, then $|\boldsymbol{p}|^{2}+|\boldsymbol{q}|^{2}<\varepsilon_{0}$. Hence for $\delta>0$ small

$$
\begin{aligned}
L(\mu, \boldsymbol{q}, \boldsymbol{p}) & =\frac{1}{2}\left[\left(\left[M^{-1}-\varepsilon^{2} \delta^{2} \Lambda\right] \boldsymbol{p}, \boldsymbol{p}\right)+(\Lambda[\boldsymbol{q}+\varepsilon \delta \boldsymbol{p}],[\boldsymbol{q}+\varepsilon \delta \boldsymbol{p}])\right] \\
& \geq-\frac{\lambda_{0}(\mu)}{2}\|\boldsymbol{q}+\varepsilon \delta \boldsymbol{p}\|^{2} \geq-\frac{\bar{\lambda}_{0}}{2}\|\boldsymbol{q}+\varepsilon \delta \boldsymbol{p}\|^{2}(1+\varepsilon \delta)^{2} \varepsilon_{0}
\end{aligned}
$$

Hence $L(\mu, \boldsymbol{q}, \boldsymbol{p})$ is bounded below if $(\mu, \boldsymbol{q}, \boldsymbol{p})$ is close to $\mathcal{M}$.

4. We have seen that the conditions guarantee that $\frac{\mathrm{d}}{\mathrm{d} t} L<0$.

So we can conclude that the manifold $\mathcal{M}$ is unstable. 
The conditions on the matrices $D_{\mu} M^{-1}(\mu) \cdot f(\mu)$ and $D_{\mu} \Lambda(\mu) \cdot f(\mu)$ are related to the curvature of the manifold $\mathcal{M}$. In theory, it would be possible for the curvature to counteract the destabilizing effects of the friction and the conditions on the matrices $D_{\mu} M^{-1}(\mu) \cdot f(\mu)$ and $D_{\mu} \Lambda(\mu) \cdot f(\mu)$ avoid this possibility.

\section{Concluding remarks}

In the examples in this paper, the dissipation is always such that the manifold of relative equilibria is invariant. In $[6,7,8]$, the influence of dissipation on stable manifolds of relative equilibria is studied and in those papers there is no need for the manifolds to be invariant under the dissipation. It would be nice if one could extend the instability results to non-invariant manifolds too. The first issue is how to define instability of a non-invariant manifold. A natural extension of the definition of instability of invariant manifolds is the following.

Definition 9 Consider the dynamical system $\dot{u}=f(u, \varepsilon)$. Let $\boldsymbol{M}_{0}$ be an invariant manifold of the system for $\varepsilon=0$. We say that $\boldsymbol{M}_{0}$ is unstable for $\varepsilon \neq 0$ if for all $K>0$ there is some $\varepsilon>0$ such that for all $\delta>0$ there is some $u_{0}$ in a $\delta$-neighborhood of $\boldsymbol{M}$ such that the solution of $\dot{u}=f(u, \varepsilon)$ with $u(0)=u_{0}$ leaves an $K \varepsilon$-neighborhood of $\boldsymbol{M}$.

This definition is the negation of the definition of stability of non-invariant manifolds. There are two differences with the definition of instability of invariant manifolds: the parameter $\varepsilon$ occurs both in the perturbation and the size of the "attracting neighbourhood". This leads to an extra factor $K$ in the definition of the "attracting neighbourhood".

The simplest example of an invariant manifold is a fixed point. A start in understanding the instability of non-invariant manifolds is the understanding of the instability of a noninvariant fixed point. If one has a fixed point of a non-degenerate Hamiltonian system and one adds a perturbation, then the implicit function theorem can be used to find a new fixed point. Furthermore, a modified Hamiltonian and a modified perturbation can be found, such that the new fixed point is invariant under the new perturbation. Chetaev's instability theorem will give the instability of this new fixed point if the perturbation is dissipative and this will lead to the instability of the original fixed point if the dissipation is sufficiently small.

However, it is not obvious how to extend this approach to manifolds of relative equilibria if the dissipation destroys the conservation laws. If the conservation law is destroyed, it is not possible to find families of relative equilibria in the dissipative system anymore. This makes it hard to get an appropriate definition for a new invariant manifold. Any set of solution curves will form an invariant manifold, but this does not seem to give a useful definition of the new invariant manifold.

In [3] and MACKAY [14], explicit expressions for the movement of the simple eigenvalues of a Hamiltonian equilibrium under dissipation can be found. From these expressions, it can be seen that the negative energy modes, i.e. the modes with negative Krein signature, will give rise to unstable eigenvalues. It is expected that a similar analysis can be done for the Lyapunov exponents of the invariant manifolds. 


\section{References}

[1] V.I. Arnold. Mathematical methods of classical mechanics (2nd ed.). Springer-Verlag, New York, 1989.

[2] T. Aubin. Nonlinear Analysis on Manifolds. Monge-Ampère Equations. Grundlehren der mathematischen Wissenschaften 252. Springer-Verlag, New-York, 1982.

[3] A.M. Bloch, P.S. Krishnaprasad, J.E. Marsden, and T.S. Ratiu. "Dissipation induced instabilities". Ann. Inst. H. Poincaré, Analyze Nonlinéaire, 11:37-90, 1994.

[4] A.M. Bloch, P.S. Krishnaprasad, J.E. Marsden, and T.S. Ratiu. "The Euler-Poincaré equations and Lie-Poisson dissipation". Comm. Math. Phys. 175:1-42, 1996.

[5] N.G. Chetaev. The Stability of Motion. Trans. by M. Nadler. Pergamon Press, New York, 1961.

[6] G. Derks, D. Lewis and T. Ratiu. "Approximations with curves of relative equilibria in Hamiltonian systems with dissipation”. Nonlinearity 8:1087-1113, 1995.

[7] G. Derks and and E. van Groesen. "Dissipation in Hamiltonian systems: Decaying cnoidal waves". SIAM Journ. of Math. Anal. 27:1424-1447,1996.

[8] G. Derks and T. Ratiu. "Attracting curves on families of stationary solutions in twodimensional Navier-Stokes and reduced magnetohydrodynamics". Proc. Roy. Soc. A, 454:1406-1444, 1998.

[9] T. Dombre, U. Frisch, J.M. Greene, M. Hénon, A. Mehr, and A.M. Soward. "Chaotic streamlines in the ABC flows". J. Fluid Mech., 167:353-391, 1986.

[10] C. Foias and J.C. Saut. "Asymptotic behavior, as $t \rightarrow \infty$ of solutions of Navier-Stokes equation and nonlinear spectral manifolds". Indiana Univ. Math. J., 33:459-477, 1984.

[11] E. van Groesen. "Time-asymptotics and the self-organization hypothesis for 2D NavierStokes equations". Physica A, 148:312-330, 1988.

[12] W. Hahn. Stability of motion. Grundlehren der mathematischen Wissenschaften in Einzeldarstellungen 138. Springer-Verlag, New York, 1967.

[13] D.D. Holm, J.E. Marsden, T. Ratiu, and A. Weinstein. "Nonlinear stability of fluid and plasma equilibria". Physics Reports, 123:1-116, 1985.

[14] R. MacKay. "Movement of eigenvalues of Hamiltonian equilibria under non-Hamiltonian perturbation". Phys. Lett. A, 155:266-268, 1991.

[15] D. Lewis, T. Ratiu, J.C. Simo, and J.E. Marsden. "The heavy top: a geometric treatment". Nonlinearity, 5:1-48, 1992.

[16] I.G. Malkin. Theory of stability of motion. Translation of Russian publication, UA Atomic Energy Commission AEC TR3352, 1952.

[17] D.R. Merkin. Introduction to the theory of stability. Texts in applied mathematics 24. Springer-Verlag, New York, 1997 
[18] J.-C. Simó, D.K. Lewis, and J.E. Marsden. "Stability of relative equilibria, Part I: the reduced energy momentum method". Arch. Rat. Mech. Anal., 115:15-60, 1991.

[19] J.-C. Simó, T.A. Posbergh, and J.E. Marsden. "Stability of relative equilibria, Part II: three dimensional elasticity". Arch. Rat. Mech. Anal., 115:61-100, 1991.

[20] R. Temam. Infinite Dimensional Dynamical Systems in Mechanics and Physics. Applied Mathematical Sciences 68. Springer-Verlag, New York, 1988.

[21] V.I. Zubov. Methods of A.M. Lyapunov and their applications. P. Noordhoff, Groningen, 1964. 